\title{
Flexible job shop scheduling using zero-suppressed binary decision diagrams
}

\author{
Meolic, R. ${ }^{a}$, Brezočnik, Z., ${ }^{a}$ * \\ ${ }^{a}$ University of Maribor, Faculty of Electrical Engineering and Computer Science, Maribor, Slovenia
}

\begin{abstract}
A B S T R A C T
A flexible job shop scheduling problem (FJSP) is a widely studied NP-hard combinatorial problem. Its goal is to optimise the production plans for simultaneously produced parts, where each part production consists of executing various operations. Each operation can be executed on several, or even all, available machines. A distinctive subproblem of FJSP is the identification of feasible solutions. A feasible solution is an allocation plan (i.e. assignment of a machine to a particular operation of a part to be produced) yielding an execution schedule satisfying the given resource constraints. FJSP is applied primarily in manufacturing systems, but it can be used to optimise Internet traffic, cloud computing, and other resource scheduling problems as well. So far, the exact methods for solving FJSP have not been considered attractive, since they seemed incapable of coping with real-size problems. This paper proposes a novel exact approach to solving FJSP which can find and count out all schedules of relatively large systems. The approach is successful due to the power of a special data structure called zero-suppressed binary decision diagrams to represent and manipulate the set of all feasible solutions efficiently. All the algorithms are implemented and tested by using our free Binary Decision Diagram package called Biddy.
\end{abstract}

\section{ARTICLE INFO}

Keywords:

Process planning;

Exact optimization;

Flexible job shop scheduling;

Unate cube set algebra;

Zero-suppressed binary decision

diagram

*Corresponding author:

zmago.brezocnik@um.si

(Brezočnik, Z.)

Article history:

Received 17 June 2018

Revised 13 November 2018

Accepted 15 November 2018

(C) 2018 CPE, University of Maribor. All rights reserved.

\section{References}

[1] Takahashi, K., Onosato, M., Tanaka, F. (2014). Comprehensive representation of feasible combinations of alternatives for dynamic production planning using zero-suppressed binary decision diagram, Journal of Advanced Mechanical Design, Systems, and Manufacturing, Vol. 8, No. 4, JAMDSM0061, doi: 10.1299/jamdsm.2014jamdsm 0061.

[2] Takahashi, K., Onosato, M., Tanaka, F. (2015). A solution method for comprehensive solution candidates in dynamic production planning by zero-suppressed binary decision diagrams, Transactions of the Institute of Systems, Control and Information Engineers, Vol. 28, No. 3, 107-115, doi: 10.5687/iscie.28.107.

[3] Phanden, R.K., Jain, A., Verma, R. (2011). Review on integration of process planning and scheduling, In: Katalinic, B., (ed.), DAAAM International Scientific Book 2011, DAAAM International, Vienna, Austria, 593-618, doi: 10.2507/daaam.scibook.2011.49.

[4] Minato, S.-I. (1993). Zero-suppressed BDDs for set manipulation in combinatorial problems, In: Proceedings of $30^{\text {th }}$ ACM/IEEE Design Automation Conference, Dallas, Texas, USA, 272-277.

[5] Minato, S.-I. (2001). Zero-suppressed BDDs and their applications, International Journal on Software Tools for Technology Transfer, Vol. 3, No. 2, 156-170.

[6] Jensen, R.A., Lauritzen, B.L., Laursen, O. (2004). Optimal task graph scheduling with binary decision diagrams, from https://pdfs.semanticscholar.org/0fe3/36b2e5e77df4ececfa749d28752694976636.pdf? ga=2.231505503. 177204796.1540581701-1029763941.1533058815, accessed May 52018.

[7] Brace, K.S., Rudell, R.L., Bryant, R.E. (1990). Efficient implementation of a BDD package. In Proceedings of the 27th ACM/IEEE Design Automation Conference (DAC '90), New York, USA, 40-45. doi: 10.1145/123186.123222. 
[8] Meolic, R., (2012). Biddy - A multi-platform academic BDD package, Journal of Software, Vol. 7, No. 6, 1358-1366, doi: 10.4304/jsw.7.6.1358-1366.

[9] Kacem, I., Hammadi, S., Borne, P. (2002). Pareto-optimality approach for flexible job-shop scheduling problems: Hybridization of evolutionary algorithms and fuzzy logic, Mathematics and Computers in Simulation, Vol. 60, No. 3-5, 245-276, doi: 10.1016/S0378-4754(02)00019-8.

[10] Kacem, I., Hammadi, S., Borne, P. (2002). Approach by localization and multiobjective evolutionary optimization for flexible job-shop scheduling problems, IEEE Transactions on Systems, Man, and Cybernetics, Part C (Applications and Reviews), Vol. 32, No. 1, 1-13, doi: 10.1109/TSMCC.2002.1009117.

[11] Chaudry, I.A., Khan, A.M., Khan, A.A. (2013). A genetic algorithm for flexible job shop scheduling, In: Proceedings of the World Congress on Engineering Vol. I, London, U.K., 703-708.

[12] Yu, M.R., Yang, B., Chen, Y. (2018). Dynamic integration of process planning and scheduling using a discrete particle swarm optimization algorithm, Advances in Production Engineering \& Management, Vol. 13, No. 3, 279-296, doi: 10.14743/apem2018.3.290.

[13] Zhang, H., Gen, M. (2005). Multistage-based genetic algorithm for flexible job-shop scheduling problem, Journal of Complexity International, Vol. 11, 223-232. 


\title{
Prilagodljivo terminiranje proizvodnje po naročilu z uporabo binarnih odločitvenih grafov s potlačenimi ničlami
}

\author{
Meolic, R. ${ }^{a}$, Brezočnik, Z. ${ }^{\text {a, }}{ }^{,}$ \\ ${ }^{a}$ University of Maribor, Faculty of Electrical Engineering and Computer Science, Maribor, Slovenia
}

\section{POVZETEK}

Problem prilagodljivega terminiranja proizvodnje po naročilu (FJSP) je široko proučevan računsko zahteven kombinatorni problem. Cilj je optimizirati proizvodni načrt za sočasno izdelavo izdelkov, pri čemer se posamezen izdelek izdela z izvedbo različnih operacij. Vsaka operacija se lahko izvede na več ali celo na vseh razpoložljivih strojih. Poseben podproblem FJSP je opredelitev primernih rešitev. Primerna rešitev je načrt razporeditve (npr. izbira stroja za izvedbo določene operacije na nekem izdelku v izdelavi), ki določa urnik izvedbe, skladen $\mathrm{z}$ omejitvami razpoložljivih virov. FJSP se v glavnem uporablja v proizvodnih sistemih, vendar se lahko uporablja tudi za optimizacijo internetnega prometa, računanje $\mathrm{v}$ oblaku in reševanje drugih problemov povezanih $\mathrm{z}$ razporejanjem virov. Natančne metode za reševanje FJSP do sedaj niso bile zanimive, saj so bile razumljene kot nezmožne spoprijeti se s problemi realnih razsežnosti. Prispevek predlaga nov, natančen pristop za reševanje FJSP, ki lahko najde in izpostavi vse razporede relativno velikih sistemov. Pristop je učinkovit zaradi sposobnosti posebne podatkovne strukture, imenovane binarni odločitveni grafi s potlačenimi ničlami, ki učinkovito predstavlja in upravlja vse primerne rešitve. Vsi algoritmi so izvedeni in testirani z uporabo naše prosto dostopne programske opreme za binarne odločitvene grafe, imenovane Biddy.

\section{PODATKI O ČLANKU}

Ključne besede:

Načrtovanje procesa;

Natančna optimizacija;

Prilagodljivo terminiranje proiz-

vodnje po naročilu;

Algebra množic kock, predstavlje-

nih s kombinacijami pozitivnih literalov;

Binarni odločitveni grafi s potlačenimi ničlami

\section{*Kontaktna oseba:}

zmago.brezocnik@um.si

(Brezočnik, Z.)

Zgodovina članka:

Prejet 17. junija 2018

Popravljen 13. novembra 2018

Sprejet 15. novembra 2018 